\title{
EL LEGADO DE SIMONE DE BEAUVOIR EN LA FILOSOFÍA FEMINISTA ESPAÑOLA
}

\author{
SIMONE DE BEAUVOIR'S LEGACY IN SPANISH FEMINIST PHILOSOPHY
}

Rosalía Romero Pérez

Universidad de Sevilla

\section{RESUMEN:}

¿Por qué hablar del legado de Beauvoir en la filosofía "feminista" y no en la filosofía? Romero inicia con esta pregunta un recorrido amplio y complejo que analiza las relaciones de El segundo Sexo, tanto en el contexto internacionalcomoenelespañol, considerando movimientos como el Sesentayochismo, y corrientes como el Feminismo Radical. Centrándose en la España del s. XXI las repercusiones de este legado y los efectos en la mujer son discutidas en distintos órdenes, moral, conocimiento y político. En ellos se trae a primer plano la contribución y los logros de mujeres en campos como la historia (transición hacia la democracia), el contexto universitario y de la filosofía académica (y sus efectos en la filosofía feminista), y las bases de una democracia basada en la paridad.

\section{PALABRAS ClAVES:}

Filosofía, Historia del feminismo, Simone de Beauvoir.

\section{ABSTRACT:}

Why should we see Beauvoir's legacy as part of "Feminist Philosophy" instead of as part of "Philosophy"? With this question Romero takes off in a wide and complex analysis of Beauvoir's legacy. Both internationally and at the level of Spain, Romero explores the relationships between The Second Sex and socio-cultural movements such as the $68^{\prime}$ and with ideologies such as Radical Feminism. Focusing on present Spain, she analyses the effects of Beauvoir's legacy as belonging to different orders, i.e. moral, knowledge and politics. In them Romero brings to the fore women's contributions and achievements in fields such as history (transition towards democracy), university context and academic philosophy (and its effects on feminist philosophy), and the basis for a democracy grounded on gender equality.

\section{KEY WORD:}

Philosophy, History of the feminism, Simone de Beauvoir. 
A MODO DE INTRODUcción

¿Por qué hablar del legado de Beauvoir en la filosofía "feminista" y no en la filosofía? La razón reside en que me referiré no sólo a la filosofía académica, ámbito en el que el uso de la expresión "Filosofía Feminista" no es unánime², sino al conjunto de ideas que inspiran a los sujetos del proceso de transformación social y político de las vidas de las mujeres, desde los últimos años de la dictadura franquista hasta la construcción de la Democracia Paritaria. Como rasgos destacados concomitantes a esta construcción de una nueva sociedad política destacaremos el notable desarrollo de los Estudios de las Mujeres, Estudios de Género e Investigaciones Feministas en las universidades españolas, así como la reticular presencia del movimiento asociativo de mujeres, organizado independientemente de los partidos políticos.

En primer lugar, es necesario hacer algunas consideraciones generales sobre el momento histórico en que se escribe y publica la obra El segundo sexo. Tales consideraciones son pertinentes desde una mirada diacrónica y evolutiva, y sería distinto si las observaciones sobre los años cuarenta, en su relación con esta obra de Beauvoir, se hicieran desde una perspectiva sincrónica. Es decir, las consideraciones a las que me voy a referir tienen importancia en virtud de lo que el paso del tiempo y los derroteros que tomó el mundo permitieron. Entre ellas señalaré que cuando Beauvoir escribe $E l$ segundo sexo había concluido la etapa sufragista, en cuyos planteamientos tuvieron prioridad la lucha por el derecho al voto femenino y el acceso de las mujeres a la educación. La sufragista y filósofa Charlotte Perkins Gilman, fallecida en 1933, había utilizado el término "androcentrismo" 3 en el título de una de sus obras, e inspirada en el darwinismo social de izquierdas consideraba que la humanidad llegaría a una etapa madrecéntrica, gracias a la supuesta existencia de una energía maternal que ella consideraba socialmente cohesionadora. Poco después de la muerte de Perkins Gilman, la faz más dura de la historia del mundo norte con su centro en Europa mostraría las serias limitaciones de las concepciones lineales y providencialistas de la historia.

Los filósofos de la Teoría Crítica de los años treinta y cuarenta se vieron sumidos en el "Pesimismo de la inteligencia", como expresara el filósofo Carlos Thiebaut ${ }^{4}$ después de conocer el genocidio nazi de los judíos, la II Guerra Mundial y la renuncia

1 Esta ponencia fue dedicada el día que la pronuncié, 29 de octubre de 2008, a mis amigas y compañeras allí presentes Nieves Pérez Gómez y Laura Villafuerte, con quienes compartí mis primeras lectura 作 de la Asamblea de Mujeres de la misma ciudad.

2 Cfr. Amorós, C., “Presentación” a Feminismo y Filosofía, Madrid, ed. Síntesis, 2000.

3 Gilman, C. P., The Man Made Word or Our Androcentric Culture, London, Fisher Unwin Press, 1979

4 Thiebaut, C., “La Escuela de Francfort”, en Victoria Camps, Historia de la Ética, vol. III, Barcelona, ed. Crítica, 1989 . del proletariado a cumplir su rol histórico: esta clase social no sólo no cumplió las predicciones de Marx sino que se hizo cómplice, en una medida desmesurada, de holocausto. Las explicaciones dadas sobre este mundo por la Primera Generación de la Escuela de Francfort supondrían un camino ya recorrido para los teóricos de la Segunda Generación de la misma escuela alemana, cuyas ideas inspiraron en muchas ocasiones a los protagonistas del Mayo del 68. Señalaré ya de entrada que, en contra de lo que se ha escrito en la prensa española en enero de 2008, en ocasión del centenario del nacimiento de Beauvoir, la autora de El segundo sexo habló de "socialismo democrático" ya en la década de los años cuarenta y fue hostil a la política que se desarrollaba en la URRS bajo el mandato de Stalin, que no moriría hasta el año 53.

El genocidio y la barbarie aludidos tuvieron una reacción institucional con una finalidad expresamente política, la Declaración Universal de los Derechos Humanos en 1948, un año antes de que nuestra filósofa publicara la obra que mostró al mundo que la mitad de la especie no era asimilada a lo genéricamente humano. En los dos tomos que dan título a El segundo sexo se realiza una apología de "la autonomía", por ser ésta un valor cuya aptitud no le era reconocida al sexo femenino; a ello sumado el lugar de "otredad" y no de Sujeto activo que las mujeres ocupan en los discursos hegemónicos de la historia del pensamiento en Occidente, desde los mitos al pensamiento racional, desde los discursos religiosos hasta los más extremadamente laicos o críticos con la cultura judeo-cristiana como, por ejemplo, lo es el psicoanálisis.

La sociedad española de estos momentos estaba inmersa en la postguerra y las feministas más destacadas del sufragismo se encontraban en el exilio. Ya habían nacido escritoras tan significativas para el feminismo y las letras españolas como Victoria Sau, Lidia Falcón; otras nacerían durante los primeros lustros de la dictadura: Mª Ángeles Durán, Celia Amorós, Victoria Sendón de León, Amelia Valcárcel, Gretel Anmann, todas ellas serían las “Hijas españolas del Sesentayochismo ${ }^{5}$ ". En la década de los años cincuenta en la filosofía académica española no era dominante el espíritu de la Institución Libre de Enseñanza, caracterizado por entender que la felicidad para las mujeres residía, en una medida considerable, en su formación educativa. Como respuesta a la publicación de El segundo sexo, Ortega y Gasset, que se exiliaría voluntariamente para volver poco después, criticaba a su autora por lo que él llamaba "manía igualitarista", y afirmaba sin paliativos que la debilidad de la mujer hace que tenga menor rango vital, y que su existir en referencia al otro es lo que la hace feliz y nos hace felices a los demás ${ }^{6}$.

5 Cfr. Romero Pérez, R.: "Hacia una historia del pensamiento feminista en España", Labrys, Revista digital de las universidades de Brasilia, Montreal y París, ed. de Ana de Miguel, monográfico dedicado al feminismo español, www. unb/br/ih/his/gefem, no 10 , dic. 2006, p. 17 y ss.

6 Ortega Y Gasset, J., El hombre y la gente, Madrid, Revista de Occidente, 1981, p. 137 y ss. 


\section{EL SESENTAYOCHISMo Y SU RECEPCIÓN EN ESPAñ}

En la década de los años sesenta emerge una conciencia crítica que se posiciona políticamente contra los totalitarismos, de derechas y de izquierdas. En Estados Unidos la New Left (Nueva Izquierda) parte de la convicción de que existe una dimensión política en la vida cotidiana. Justamente esta convicción posibilitará el nacimiento de los grupos de feministas radicales en Estados Unidos, concretamente en Nueva York y en Chicago. Entre sus reivindicaciones tenían prioridad el derecho a los anticonceptivos, el derecho al divorcio, el derecho al aborto, a una legislación que protegiera a las mujeres de la violencia doméstica... ${ }^{7}$ al mismo tiempo que se denunciaban los estereotipos sexistas vehiculados por los medios de comunicación Como la filósofa italiana Lidia Cirilo ha expresado, el feminismo se convirtió en un movimiento de masas en virtud del Sesentayochismo ${ }^{8}$.El Sesentayochismo es un acontecimiento, o cúmulo de acontecimientos generados en torno del "Mayo del 68 francés", que afectará tanto al orden político como al orden del conocimiento. En ambos casos ha de ser re-pensado el "Sujeto". En el orden de lo político nace la idea de un sujeto de transformación social fragmentado; las minorías, en el sentido de grupos o colectivos carentes de poder, comienzan a ser objeto del pensamiento. En el orden del conocimiento se genera una renovación de las categorías fundamentales de la ciencia y la teoría occidentales. En este orden, el pensamiento sobre el Sujeto o los Sujetos del conocimiento es una cuestión con una proyección ulterior de dimensiones inimaginables para la sociedad de aquél entonces. Para una minoría como el colectivo de las mujeres, un nuevo sujeto emergente, y para la crítica al androcentrismo del sujeto del conocimiento existía una obra sin precedentes, El segundo sexo de Simone de Beauvoir. La amplitud de ideas y corrientes del Sesentayochismo y del Feminismo Radical ayuda a explicar su éxito y su expansión; a grandes rasgos se generan dos líneas básicas de pensamiento y de actuación: la pro-institucional y la contracultural. La obra de Beauvoir dará luz y agua a ambas corrientes. Entre las militantes pertenecientes a los primeros grupos de feministas radicales destacan Kate Millett y Shulamith Firestone, autoras respectivamente de dos obras clásicas del Feminismo Radical: Política sexual y La dialéctica del sexo, esta última dedicada a Beauvoir. En ambas el legado de El segundo sexo es patente, hasta tal punto que Cèlia Amorós ha expresado que todo lo que se ha hecho en Teoría Feminista después de Beauvoir no son sino notas a pie de página de $E l$ segundo sexo.

En los primeros años de la década de los setenta, en España crecía la lucha antifranquista y los acontecimientos del exterior se hacían sentir tanto en el mundo

7 Echols, A., Daring to be bad. Radical Feminismi in America. 1967-1975, University of Minnesota Press, 1989.

8 Cirilo, L., “Una mujer en el 68. Entrevista a Lidia Cirillo”, en Inprecor, noำ61, 1988, p. 35. obrero como en el ámbito universitario. Las emisoras de radio jugaron un papel destacado en la recepción de lo que ocurría fuera de nuestras fronteras territoriales: la BBC de Londres y la Pirenaica impregnaban de alegría y optimismo a quienes pegaban la oreja cada noche a su radio, pendiente siempre de quién llamaba a la puerta. En Europa se hablaba de la crisis de la dictadura franquista española y la prensa nacional se hacía eco de noticias importantes en el espacio internacional. Entre ellas citaremos la entrevista realizada por un periodista norteamericano a la Primera Ministra de Israel, publicada en el ABC dominical en noviembre de 1971. Destacaremos que ante la pregunta sobre la compatibilidad del cargo que ocupaba la entrevistada y su sexo Golda Meir respondió que era una responsabilidad que afectaba negativamente al corazón, pero al corazón de hombres y de mujeres. Por esta entrevista supimos también que esta gobernante dio una respuesta inédita a la propuesta de un ministro fundamentalista religioso, que pretendía que las mujeres en Israel no salieran por la noche, para así evitar las agresiones masculinas: Golda Meir respondió que el toque de queda al anochecer habría que dictarlo para los agresores, para quienes eran los causantes del problema de la violencia contra las mujeres 9.

En la lucha por el derecho al aborto en Francia se emitió un comunicado público en 1973, firmado por más de trescientos médicos/as, entre quienes figuraban tres Premios Nobe ${ }^{10}$. El Movimiento Feminista francés tuvo una notable presencia y Simone de Beauvoir era una relevante figura. En España, prohibidos los métodos anticonceptivos, el número de interrupciones de embarazo clandestinas, en manos de personal no cualificado para ello, provocaba cada año numerosísimas muertes. Según los datos que se manejaban en el Movimiento Feminista en 1985, cuando el Partido Socialista despenalizó parcialmente el aborto, cada año morían 3000 españolas por interrumpir sus embarazos en condiciones infrahumnas; 3000 españolas pobres, claro está. Quienes tenían recursos acudieron, como consta en sus registros, a las clínicas de Amsterdam y de Londres.

En este contexto, la Organización de Naciones Unidas declara 1975 el Año Internacional de la Mujer. El caldo de cultivo gestado en nuestras tierras permitió que Victoria Sau publicara en 1974 el extraordinario Manifiesto para la Liberación de la Mujer. En esta obra, en cuyas páginas aparece el nombre de Simone de Beauvoir, su autora denuncia la desigualdad entre los sexos y llama a la politización y a la organización de grupos de mujeres, con el objetivo de encauzar su propia lucha. Del extraordinario contenido expuesto y argumentado en las páginas de este Manifiestodestacaremos las que Victoria Sau hace sobre "el poder": afirma que el poder no hay que entenderlo

9 ABC, suplemento dominical 5-12-1971.

10 Macey, D., Las vidas de Michel Foucault, trad. cast. de Carmen Martínez Gimeno, Madrid, eds. Cátedra, 1993, p. 396. 
como una estructura piramidal, no consiste exclusivamente en llegar al más alto cargo es más, mujeres excepcionales ha habido siempre y la representación femenina, a mínimos niveles, es "autorizada y consentida por los hombres con frecuencia para disimular, a modo de pantalla, la realidad interior, mucho menos halagüeña"11. Victoria Sau defiende que el poder está en las tareas anónimas de cada día: ser profesor de Universidad, ser miembro del consejo de Administración de una empresa, ser concejal, diputado, juez, cirujano, director de Banco, de periódico, de cárcel, de reformatorio.. V. Sau llama la atención sobre el hecho de que en ningún país del mundo las mujeres comparten el 50\% del poder que como mitad de la especie humana les corresponde. Se informa también de los interesantísimos movimientos feministas, incluidos algunos en los que también participaban hombres, habidos en Estados Unidos y Europa: Los Ángeles, Chicago, Nueva York, Washington, Noruega, Dinamarca, Finlandia...

El movimiento feminista organizado en los últimos años de la dictadura y primeros de la transición marcó, no obstante, unas prioridades muy en concordancia con las reivindicaciones originarias del Feminismo Radical en Chicago y Nueva York, extendidas por todo el mundo occidental: derecho al uso de anticonceptivos, derecho al divorcio, derecho al aborto y todo un cúmulo de reivindicaciones legales necesarias para sacar a las mujeres de la "minoría de edad" legal, y para sacarlas de las cárceles, donde se encontraban unas dos mil solamente por delito de adulterio. Las reivindicaciones que señalamos no están puestas al azar, lo que quiere decir que no estamos nombrando muchas otras del mismo modo importantísimas; pero vamos al tema que nos ocupa: la conexión con Simone de Beauvoir.

En estos momentos de reconstrucción del sistema democrático español teníamos muchas maestras, las nacidas durante la II República y en la postguerra, anteriormente citadas. E incluso había madres, resultantes de distintas interpretaciones de la obra de Beauvoir, que dieron lugar a distintas corrientes del Movimiento Feminista. Y es más, en España tomamos como maestras del pensamiento feminista no sólo a las españolas sino también a teóricas y militantes como Kate Millet, Shulamith Firestone, Christine Delphy, Rossana Rosanda, por citar algunas de las más destacadas, cuyos libros fueron leídos, y en ocasiones debatidos en los grupos de estudio. ¿Qué representa, entonces, Simone de Beauvoir? Simone de Beauvoir es una referencia. El segundo sexo es un tratado de biología, psicoanálisis, marxismo, historia, mitología, en lo que respecta al tomo "Los hechos y los mitos"12, donde se muestra el androcentrismo del saber hegemónico. En el tomo "La experiencia vivida"13 parte del principio existencialista

11 Sau, V., Manifiesto para la liberación de la mujer, Barcelona, eds. 29, 1974, p. 283.

12 De Beauvoir, S., El segundo sexo. T. I. Los hechos y los mitos, trad. cast. Pablo Palant, Buenos Aires, ed. Siglo Veinte, 1987

13 De Beauvoir, S., El segundo sexo. T. II. La experiencia vivida, trad. cast. Pablo Palant, Buenos Aires, ed. Siglo Veinte, 1987. "la existencia precede a la esencia" y explica cómo llegamos a ser mujeres. A Beauvoir le tocó explicar que para las mujeres la maternidad no es inexorablemente "destino", a diferencia de lo que defendía Perkins Gilman en la primera mitad de siglo, sino que la relación lógica de implicación establecida entre ser mujer y ser madre es una de las creencias ancestrales de las sociedades de dominación masculina ${ }^{14}$. Beauvoir analiza la condición social de las mujeres interdisciplinar y multidisciplinarmente ${ }^{15}$.

En todas las disciplinas del saber a las que Beauvoir aplicó la crítica se ha introducido su perspectiva crítica. El concepto de "androcentrismo" en el pensamiento feminista entra en otra etapa: si para Perkins Gilman hacía referencia a la sociedad gobernada por los padres, a partir de Beauvoir el concepto de "androcentrismo" se resignificará para referirse a la exclusión de las mujeres del Sujeto del conocimiento y, por extensión analítica, de lo concebido como lo genéricamente humano.

\section{EL LEGADo DE SIMoNe DE BEAUVoIR EN LA ESPAña DEL SIGLo XXI}

La recepción en España de la obra de Beauvoir tiene una coloración propia, a juzgar por la evolución de las mujeres en la etapa de la transición, el desarrollo de los Estudios no-androcéntricos, la importancia que se le reconoce en el seno de la filosofía académica y la construcción de la democracia paritaria. Veamos cada uno de estos aspectos:

a) La transición que las mujeres tuvieron que hacer desde una dictadura militar con un Estado confesional, católico, apostólico y romano a una sociedad democrática. El aspecto al que vamos a referirnos afecta al orden de la moral. Para comprender esta transición hemos de recordar que estamos hablando del paso de un "patriarcado de coerción" a un "patriarcado de consentimiento", conceptualización establecida por Alicia Puleo ${ }^{16}$. Mientras que los "patriarcados de coerción" prohíben mediante la violencia o la fuerza coercitiva, en los "patriarcados de consentimiento" se seduce a las mujeres para que lleguen a desear parecerse a los modelos presentados en la publicidad y otros vehículos socializadores.

Muchas mujeres españolas, militantes feministas y mujeres que no lo eran, se vieron identificadas con Beauvoir en la ruptura con la moral católica; aunque Francia es un país laico su educación se forjó en el seno de una familia fundamentalmente católica,

14 Esta temática fue posteriormente desarrollada por su discípula Elisabeth Badinter en su obra L'amour en plus. Histoire de l'amour maternal (XVIIe-XX siècle), Paris, Flammarion, 1980. Hay trad. cast. de Marta Vasallo: ¿Existe el instinto maternal? Historia del amor maternal. Siglos XVII al XX ed. Paidós, 1991. 15 Romero Pérez, R., “La familia filosófica de Simone de Beauvoir” en Amelia Valcárcel y Rosalía Romero Pérez (eds.), Pensadoras del sigo XX, Sevilla, ed. Instituto Andaluz de la Mujer, col.
"Hypatia", 2001, pp. 78-79. 16 Puleo, A., Filosofía, género y pensamiento crítico, Publicaciones de la Universidad de Valladolid col. "Acceso al saber", 2000, p. 37 
con lo que tuvo que romper cuando, siendo una adolescente, se pronunció a favor del uso de los anticonceptivos, del aborto, del divorcio, todos ellos temas que rompían con las prescripciones morales establecidas por el fundamentalismo católico. En España comienza un proceso de laicización de la sociedad o secularización de la moral justamente con la transición democrática, y un referente de primer orden para muchas mujeres es Simone de Beauvoir, precisamente por la identificación con la historia de vida que nuestra autora cuenta en su obra literaria, concretamente en Memorias de una joven formal.

b) El desarrollo de los Estudios de las Mujeres, Estudios de Género o Investigaciones Feministas en las universidades españolas. Este aspecto afecta al orden del conocimiento. En 1976 Cèlia Amorós publica un artículo en el que realiza una apología del pensamiento de Simone de Beauvoir ${ }^{17}$, trabajo que será posteriormente uno de los capítulos de su obra Hacia una crítica de la razón patriarcal ${ }^{18}$, donde en sus primeras páginas recuerda la influencia que Beauvoir había tenido en la trayectoria intelectual de Jean-Paul Sartre. Después de la publicación de El segundo sexo los personajes femeninos de las obras de teatro de Sartre son presentados como portadores de moral, a diferencia de la identificación de lo femenino con lo viscoso encontrada en El ser y la nada. Cèlia Amorós plantea la necesidad de que en el ámbito institucional, universitario, se reciba esta aportación de la filósofa francesa y señala el androcentrismo que impregna a la institución universitaria española. La comprensión interdisciplinar y multidisciplinar de la condición de las mujeres beauvoiriana tiene un impacto visible: señalaremos la importante función que han desempeñado y desempeñan los "Seminarios Interdisciplinares de Estudios de la Mujer", creados en principio en algunas universidades y reproducidos por toda la geografía española. No sólo en el espacio de la Academia y de la Universidad, sino en las actividades formativas desarrolladas en el seno de los Movimientos Feministas se ha tomado en numerosas ocasiones la iniciativa de organizar Seminarios, Encuentros, Jornadas, también con ese carácter multidisciplinar e interdisciplinar que Beauvoir mostró necesario realizar para tener una visión global de la condición del segundo sexo.

c) La filosofía académica. Este aspecto afecta al orden del conocimiento y al desarrollo de la filosofía feminista o feminismo filosófico. Las herederas legítimas de Beauvoir en la filosofía española son Cèlia Amorós y Amelia Valcárcel, quienes desde sus tempranas juventudes se dedican a la filosofía y al feminismo. Si bien Cèlia Amorós considera que no se puede hablar de Filosofía Feminista del mismo modo que

17 Amorós, C., “¿Feminismo existencialista versus feminismo estructuralista? Notas para una reflexión sobre la crítica de Juliet Mitchell a Simone de Beauvoir", Negaciones, nํㅡ 2, diciembre 1976, pp. 25-38.

18 Amorós, C., Hacia una crítica de la razón patriarcal, Barcelona, ed. Anthopos, 1985. se habla, por ejemplo, de Filosofía Analítica, destacaremos que las hijas de Beauvoir en España se enmarcan en lo que se ha llamado "Feminismo Ilustrado". Amorós creó el "Seminario Permanente Feminismo e Ilustración" en la Universidad Complutense de Madrid, y se reunió durante cinco años, de los que dan fe el conjunto de trabajos publicados en sus Actas $^{19}$; posteriormente Cèlia Amorós ha continuado con otros grupos de trabajo, si bien el núcleo de investigadoras inicial ha permanecido, en el que destaca por sus investigaciones y publicaciones sobre Beauvoir Teresa López Pardina ${ }^{20}$ Amelia Valcárcel, por su parte, y en estrecha colaboración con la Escuela de Amorós, ha desarrollado una extraordinaria obra ${ }^{21}$ en la que la Ilustración con todo su aparato conceptual tiene un lugar nuclear ${ }^{22}$. Si bien en estas líneas es difícil sintetizar lo más importante de la herencia de Beauvoir en las obras de estas pensadoras, lo que exigiría un trabajo específico y pormenorizado, voy a introducirme en aspectos como la crítica al naturalismo y al esencialismo y en la dialéctica del amo y del esclavo.

En lo que se refiere al pensamiento de Amorós y su comprensión nominalista del patriarcado, entendido como un sistema de reparto asimétrico de poderes, en la línea crítica beauvoiriana de lo "femenino", la que ha sido la primera mujer en España que ha recibido el Premio Nacional de Ensayo, después de 30 años de existencia de este galardón, ha trasladado el debate de los "universales" de la Baja Edad Media a la actualidad. Para explicar que la "feminidad" no es ni una esencia ni una expresión de la naturaleza, en su trabajo "A vueltas con el problema de los universales: Guillerminas, Roscelinas y Abelardas" ${ }^{23}$ su autora reproduce el debate en torno a las obras de Platón y Aristóteles: la feminidad puede entenderse ante-rem, comprensión esencialista platónica que defiende que la esencia pre-existe a lo concreto y material con lo cual lo femenino pre-existiría a las mujeres. Otra comprensión distinta sería en torno al universal in rem: aquí reside la comprensión aristotélica, la cual defiende que el universal no está separado del individuo concreto, sino que forma parte del mundo físico, de la naturaleza, y es constitutivo del singular, lo que significa que la feminidad sería una expresión de la naturaleza. Y, por último, expone la comprensión nominalista

19 Amorós, C. (coord..), Actas del Seminario Permanente "Feminismo e Ilustración" (1988-1992) Madrid, ed. Instituto de Investigaciones Feministas de la Universidad Complutense y Dirección General de la Mujer de la Comunidad de Madrid, 1992.

20 Entre sus trabajos citaremos López Pardina, T., Simone de Beauvoir. Una filósofa del siglo XX Publicaciones de la Universidad de Cádiz, 1998, y Simone de Beauvoir (1908-1986), Madrid, Ediciones del Orto, col. "Biblioteca Filosófica", 1999.

21 Sobre la filosofía de esta pensadora puede verse Rosalía Romero Pérez, Amelia Valcárcel, Madrid eds. del Orto, col. "Biblioteca de Mujeres", 2003.

22 De su obra feminista puede verse Valcárcel, A., Sexo y Filosofía. Sobre "mujer" y "poder". Barcelona, ed. Anthropos, 1991, y La política de las mujeres, Madrid, ed. Cátedra, col. "Feminismos", 1997.

23 Amorós, C., "A vueltas con el problema de los universales: Guillerminas, Roscelinas y Abelardas" en La gran diferencia y sus pequeñas consecuencias para las luchas de las mujeres, Madrid, ed. Cátedra, col. "Feminismos", 2005, pp. 73-85. 
que defiende que el universal no antecede al individuo ni es parte constitutiva de él sino que el universal está en la mente, sería el universal post-rem.

d) La fundamentación de la democracia paritaria. Este aspecto afecta al orden político, si bien se inmiscuye en lo social y afecta a todas las instituciones públicas. Si bien Victoria Sau en su Manifiesto planteó la exclusión de las mujeres de los puestos de poder que como mitad de la especie les corresponden, la fundamentación de la democracia paritaria en España ha requerido un intenso trabajo de abstracción teórica e incluso ha generado enfrentamientos en el seno del Movimiento Feminista. El "Cisma de Granada" en 1979 se produjo a partir del debate sobre "doble militancia" (en el Movimiento Feminista y en Partidos políticos) o "militancia única" (en el Movimiento Feminista). En esta última posición se encontraba la filósofa Victoria Sendón de León, en cuya obra desarrolla una crítica a Beauvoir en torno a sus raíces hegelianas ${ }^{24}$. La fundamentación de la Democracia Paritaria ha corrido a cargo de las feministas de la Igualdad y a favor de la doble militancia, planteando en sus propios partidos políticos la cuestión de la paridad; estamos hablando de organizaciones políticas de izquierda porque las de derecha se han opuesto tajantemente siempre a la paridad, si bien en algún momento tendremos que explicar cómo ha influido la reivindicación de la paridad en los partidos de derecha. Ello es necesario porque si bien no asumieron el planteamiento paritario sí que lo integraron en su super-yo, dando como resultado el creciente número de mujeres en sus organizaciones. En la fundamentación teórica que las feministas de la Igualdad han llevado a cabo, a partir de la década de los años ochenta en España, son insoslayables los nombres de las "Hijas de Beauvoir": Amorós y Valcárcel. En el telón de fondo de sus respectivas posiciones político-feministas subyace la explicación beauvoiriana sobre el origen de las sociedades de dominación masculina. La argumentación reside en la exclusión de las mujeres de las expedicione guerreras, en las que se las consideraba intrusas por entender que su función natural era la reproducción de la especie, en las sociedades primitivas, en las que poder militar y poder político eran una misma $\operatorname{cosa}^{25}$. La actual ministra Doña Carmen Chacón nombrada Ministra de Defensa cuando estaba embarazada, es la expresión flagrante del desafío a la tradicional dominación masculina analizada en las páginas de la obra de Beauvoir.

Voy a explicar en esta última parte la herencia beauvoiriana que se encuentra en la filosofía política de Amelia Valcárcel para comprender uno de sus planteamientos iniciales, que generaron una amplia polémica en los primeros años de los ochenta. Valcárcel plantea que a las mujeres no se les debe exigir más que a los varones para que ocupen puestos de poder; lógicamente esto supondría una discriminación añadida. Por

24 Sendón De León, V., Marcar las diferencias, Barcelona, ed. Icaria, 2002, p. 23.

25 De Beauvoir, S., El segundo sexo. T. I. Los hechos y los mitos, op. cit., pp. 87-88. ello critica a los teóricos que integran a las mujeres en comprensiones soteriológicas y les atribuyen, por adelantado, el rol de "salvadoras" de todos los males que no han sabido resolver los varones que han detentado el poder político u otros poderes. Y por ello Valcárcel plantea "El derecho al mal”. Esta reivindicación supone no sólo frenar una discriminación añadida ad hoc sino que es necesario que se comprenda a raíz del propio concepto de emancipación de Valcárcel, en el que se encuentran profundas raíces de los análisis de El segundo sexo.

Beauvoir interpreta a Hegel desde su concepción existencialista propia: la dialéctica del amo y del esclavo, expuesta en la Fenomenología del espíritu, es una lucha por el reconocimiento, lo que significa que es una lucha inevitable en cualquier sociedad humana, en tanto nos hacemos humanos cuando los otros nos reconocen y reconocemos a los demás. Pero que sea inevitable no significa que este esquema sea estático, sino por el contrario es dinámico, dialéctico y como tal las posiciones del amo y del esclavo son situacionales. Es decir, que en el momento en que se desplace el esclavo de su situación, el amo, por efecto correlativo, queda des-situado, ya no es el amo, o no es el mismo amo, ha habido necesariamente cambio en su autoconciencia y, por consiguiente, su identidad ha quedado alterada, desplazada.

El reconocimiento es, por tanto, una de las cuestiones principales de la formación de cualquier orden social humano; el patriarcado no es una excepción: los grupos sexuales existen desde el momento en que se reconocen como tales y aceptan las supuestas diferencias. Si aplicamos a las mujeres el mismo proceso que al esclavo hegeliano, naturalmente, si la mujer se des-sitúa de su condición femenina, y todo lo que ello conlleva, tiene un efecto correlativo sobre la situación que define provisionalmente la identidad del colectivo de los varones, y éste es un buen método para erradicar las relaciones jerárquicas entre los grupos sexuales. Toda esta comprensión beauvoiriana es necesaria para ver cómo Amelia Valcárcel entiende la emancipación de las mujeres: des-situarse como esclavo, como mujer, puede incluir adoptar aspectos de la situación del amo, lo que en sí mismo es emancipatorio porque lo que se mide es si la existencia del dominante ha sido socavada como dominador: el cambio de la posición del grupo de los varones como efecto correlativo de las nuevas posiciones de las mujeres. De ahí que Amelia Valcárcel reivindique el derecho al mal.

Para concluir, recordaremos que Simone de Beauvoir no era consciente de la magnitud y, sobre todo, de la grandeza de su esfuerzo cuando escribió El segundo sexo. Quizás cuando murió en 1986 tampoco lo sabía. Su trabajo no tuvo la voluntad de sistema y, por ello, es lógico que algunas problemáticas del colectivo de las mujeres deban ser abordadas recurriendo a otras fuentes. A ello sumado algo esencial: como hemos visto el mundo en que fue escrito y recepcionado El segundo sexo dista de mundo de Hoy, resultado de un acelerado proceso de cambio en los últimos lustros 
del siglo XX y primeros años del siglo XXI. Aun así no podemos negar que, a favor o en contra de las posiciones filosóficas y políticas de Beauvoir, habitamos un mundo postbeauvoiriano, en el sentido de que es imposible pensar el presente y el futuro sin la autora de El segundo sexo. Habitamos un mundo post-beauvoiriano y es deseable que habitemos un planeta con la misma cualidad. ¿Sería posible la liberación real de las mujeres de las distintas culturas sin cuestionar sus sistemas de conocimiento y de creencias? ¿Sería posible terminar con la violencia contra las mujeres sin cuestionar los discursos que la legitiman? ¿Sería posible que las mujeres de las distintas sociedades del planeta avanzaran hacia la igualdad sin que se les reconociese su acción en la cultura y se eliminase el veto de participación en los Sujetos hegemónicos?

\section{BIBLIOGRAFÍA}

Amorós, C. (1976) ¿Feminismo existencialista versus feminismo estructuralista? Notas para una reflexión sobre la crítica de Juliet Mitchell a Simone de Beauvoir, Negaciones, $n^{\underline{0}}$ 2, diciembre.

Amorós, C. (1985). Hacia una crítica de la razón patriarcal. Ed. Anthopos: Barcelona.

Amorós, C. (1992), Actas del Seminario Permanente "Feminismo e Ilustración" (19881992), Madrid, ed. Instituto de Investigaciones Feministas de la Universidad Complutense y Dirección General de la Mujer de la Comunidad de Madrid.

Amorós, C. (2000). "Presentación" a Feminismo y Filosofía. Síntesis: Madrid.

Amorós, C. (2005). A vueltas con el problema de los universales: Guillerminas, Roscelinas y Abelardas, en La gran diferencia y sus pequeñas consecuencias para las luchas de las mujeres. Ed. Cátedra, col. "Feminismos": Madrid.

Badinter, E. (1980). L'amour en plus. Histoire de l'amour maternal (XVIIe-XX siècle). Flammarion: Paris.

Cirilo, L. (1988). Una mujer en el 68. Entrevista a Lidia Cirillo, en Inprecor, no 61.

De Beauvoir, S. (1987) El segundo sexo. T. I. Los hechos y los mitos, trad. cast. Pablo Palant.Ed . Siglo Veinte: Buenos Aires.

Echols, A. (1988). Daring to be bad. Radical Feminismi in America. 1967-1975, University of Minnesota Press: Minnesota.

Gilman, C. P. (1979). The Man Made Word or Our Androcentric Culture. Fisher Unwin Press: London.

López Pardina, T. (1998) Simone De Beauvoir. Una filósofa del siglo XX. Publicaciones de la Universidad de Cádiz: Cádiz.

López Pardina, T. (1999). Simone de Beauvoir (1908-1986), Ediciones del Orto, col. "Biblioteca Filosófica": Madrid.

Macey, D. (1993). Las vidas de Michel Foucault, trad. cast. de Carmen Martínez Gimeno. Eds. Cátedra: Madrid.

Ortega, Y; Gasset, J. (1981). El hombre y la gente. Revista de Occidente: Madrid.

Pérez, R. (2001). La familia filosófica de Simone de Beauvoir en Amelia Valcárcel y Rosalía Romero Pérez (eds.), Pensadoras del sigo XX. Ed. Instituto Andaluz de la Mujer, col. "Hypatia": Sevilla.

Pérez, R. (2006). Hacia una historia del pensamiento feminista en España , Labrys, Revista digital de las universidades de Brasilia, Montreal y París. Ed. de Ana de Miguel, monográfico dedicado al feminismo español, www. unb/br/ih/his/gefem, $\mathrm{n}^{-}$10, dic. 2006, p. 17 y ss. 